\title{
Impact of Overt Hypothyroidism on Early Outcomes of Coronary Artery Surgery
}

\author{
Dong Zhao, MD,${ }^{1}$ Wei Feng, MD,${ }^{1}$ Wei Zhao, $\mathrm{MD},{ }^{2}$ Xin Yuan, $\mathrm{MD},{ }^{1}$ Chuangshi Wang, $\mathrm{PhD}{ }^{3}$ \\ ${ }^{1}$ Department of Adult Cardiac Surgery, Fuwai Hospital, National Center of Cardiovascular Diseases, Chinese Academy of Medical \\ Sciences and Peking Union Medical College, Beijing, China; ${ }^{2}$ Department of Information, Fuwai Hospital, National Center of \\ Cardiovascular Diseases, Chinese Academy of Medical Sciences and Peking Union Medical College, Beijing, China; ${ }^{3}$ Medical \\ Research and Biometrics Center, Fuwai Hospital, National Center of Cardiovascular Diseases, Chinese Academy of Medical Sciences \\ and Peking Union Medical College, Beijing, China
}

\section{ABSTRACT}

Background: The association between preoperative overt hypothyroidism and early outcomes after coronary artery bypass grafting $(\mathrm{CABG})$ is unclear. This study aimed to evaluate the influence of overt hypothyroidism on the outcomes of CABG.

Methods: The series included 189 overt hypothyroid patients, who underwent CABG at Fuwai Hospital. These patients were 1:4 matched with 737 euthyroid patients using propensity score matching. The early postoperative outcomes were compared.

Results: After propensity score matching, the incidences of impaired wound healing, reintubation, and the total complications were higher in hypothyroid patients than euthyroid patients $(11.8 \%$ vs. $0.9 \%, P<0.001 ; 2.1 \%$ vs. $0.4 \%, P=0.03$; $39.6 \%$ vs. $30.3 \%, P=0.015$, respectively). Multivariate analysis showed overt hypothyroidism was significantly associated with the occurrence of impaired wound healing (odds ratio $[\mathrm{OR}]=12.29, P<0.001)$, reintubation $(\mathrm{OR}=5.71, P=0.047)$, and the total complications $(\mathrm{OR}=1.31, P=0.049)$. The $\mathrm{OR}$ of the total complications was $1.43(P=0.03)$ in hypothyroid patients with abnormal thyroid-stimulating hormone compared with euthyroid patients. The proportions of the use of dopamine, adrenaline, milrinone, and dobutamine in hypothyroid patients were higher than euthyroid patients $(75.4 \%$ vs. $67.6 \%, P=0.038 ; 10.7 \%$ vs. $6.1 \%, P=0.028 ; 3.2 \%$ vs. $0.3 \%, P=0.001 ; 4.8 \%$ vs. $1.2 \%, P=0.004$, respectively). The total duration of inotropic support and mechanical ventilation time in hypothyroid patients were longer than euthyroid patients (median duration: 4 days vs. 3 days, $P=0.003 ; 17$ hours vs. 15 hours, $P<0.001$, respectively).

Conclusions: CABG in overt hypothyroid patients is associated with a higher incidence of postoperative complications, stronger postoperative inotropic support, and longer mechanical ventilation time.

Received fune 18, 2021; accepted fuly 9, 2021.

Correspondence: Wei Feng, MD, Department of Adult Cardiac Surgery, Fuwai Hospital, National Center of Cardiovascular Diseases, Chinese Academy of Medical Sciences and Peking Union Medical College, 100037, No. 167 North Lishi Road, Xicheng District, Beijing, China, Telephone +86-01088322355 (e-mail: fengwei@fuwai.com).

\section{INTRODUCTION}

The thyroid function influences every structure of our body. Thyroid hormones have direct effects on cardiovascular function and indirect effects mediated through the autonomic nervous system, vascular compliance, vasoreactivity, and renal function [Grais 2014]. The prevalence of hypothyroidism is between 1\% and 17\% [Okosieme 2016; Shan 2016]. Overt hypothyroidism is a more severe stage than subclinical hypothyroidism and is accompanied by increased serum thyroid-stimulating hormone (TSH), decreased thyroxine, and symptoms. Overt hypothyroidism is associated with impaired cardiac contractility, decreased cardiac output, increased systemic vascular resistance, accelerated atherosclerosis, and increased risk of coronary artery disease [Biondi 2012; Grais 2014; Kinugawa 2001; Klein 2007]. Patients with severe coronary diseases who underwent coronary artery bypass grafting (CABG) are at high risk of adverse cardiovascular events. The postoperative recovery of these patients may be complicated by preoperative overt hypothyroidism. However, limited data are available on the relationship between preoperative overt hypothyroidism and early outcomes after CABG, most of which were limited by small sample sizes. This study aimed to evaluate the influence of overt hypothyroidism on the recovery of patients after CABG by using a propensity score matching method.

\section{METHODS}

From January 2015 to December 2018, 189 patients with overt hypothyroidism who underwent CABG at Fuwai Hospital (Beijing, China) were identified. Overt hypothyroidism was defined as a documented history of overt hypothyroidism in the patients' clinical record. The exclusion criteria of the overt hypothyroid group included (1) no thyroid function tests within two weeks before surgery; (2) patients with subclinical hypothyroidism; (3) concurrent with other cardiac surgery; and (4) previous sternotomy. (Figure 1) The exclusion criteria of the euthyroid group were (1) no thyroid function tests within two weeks before surgery; (2) abnormal thyroid function results; (3) history of thyroid or pituitary disease; (4) concurrent with other cardiac surgery; and (5) previous sternotomy. Finally, 6718 euthyroid patients were identified. The overt hypothyroid patients were then matched 1:4 with 


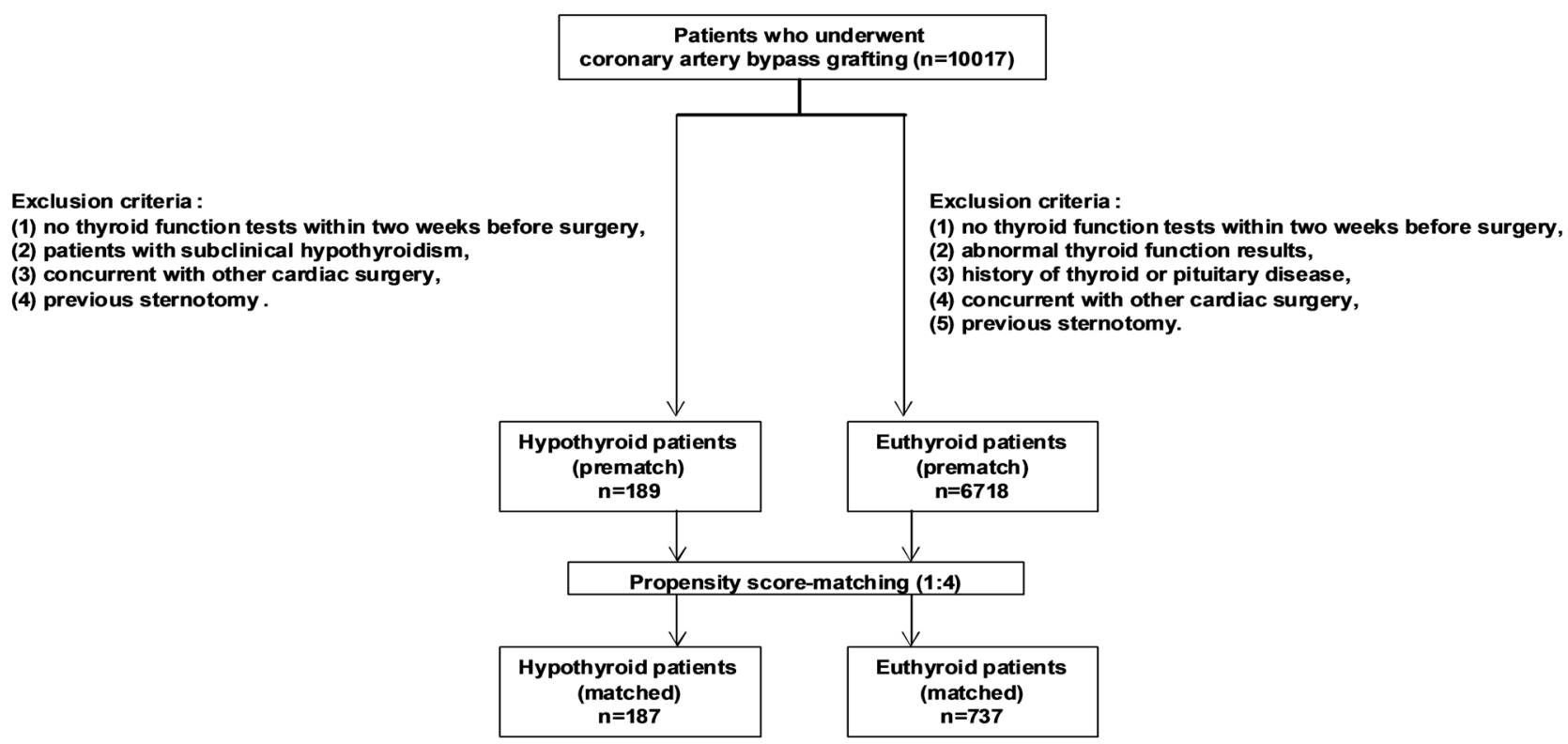

Figure 1. Flow chart of patient selection.

euthyroid patients. Medical history and perioperative records were collected. Echocardiography was used to measure left ventricular end-diastolic diameter (LVEDD) and left ventricular ejection fraction (LVEF). Because this was a retrospective analysis of data collected for routine clinical care, individual informed consent was waived by the ethics committee. The study design was approved by the local ethics committee of Fuwai Hospital, Beijing, China (Approval NO. 2019-1151; Date of review: June 26, 2019).

Median sternotomy is the preferred approach in all patients. The decision to use cardiopulmonary bypass (CPB) was left to the operating surgeon and mostly was based on the severity and extent of disease of the target vessels. CPB was conducted on moderate hypothermia, and myocardial protection was achieved by using intermittent anterograde hyperkalemic cold blood cardioplegia. The final goal of CABG was to obtain complete myocardial revascularization.

For thyroid function tests, serum TSH, free thyroxine (FT4), total thyroxine (TT4), free triiodothyronine, and total triiodothyronine were measured using electrochemiluminescence immunoassays on a Siemens analyzer. Euthyroidism was defined as $0.55 \mathrm{uIU} / \mathrm{mL} \leq$ TSH level $\leq 4.78 \mathrm{uIU} / \mathrm{mL}, 0.8$ $\mathrm{ng} / \mathrm{dL} \leq \mathrm{FT} 4 \leq 1.88 \mathrm{ng} / \mathrm{dL}$ and $4.29 \mathrm{ug} / \mathrm{mL} \leq \mathrm{TT} 4 \leq 12.47$ $\mathrm{ug} / \mathrm{mL}$ at the time of surgery, and no documented history of thyroid disease in the patient's clinical record.

The primary outcome was postoperative complications, which included impaired wound healing, reintubation, tracheotomy, reoperation, thoracentesis or pericardiocentesis, intra-aortic balloon pump (IABP) implantation, dialysis, postoperative stroke, pacemaker implantation, ventricular arrhythmia, atrial fibrillation, and death. The impaired wound healing was defined as hematoma, wound dehiscence, skin necrosis, lymphatic wound drainage, and local signs of infection. Postoperative stroke was defined as a new neurological deficit with imaging of the central nervous system infarction or hemorrhage. Reoperation was defined as the second operation under general anesthesia, during the same hospitalization. Patients routinely were monitored postoperatively for the occurrence of arrhythmia by bedside monitors. When needed, 12-lead electrocardiograms were obtained to determine the exact cardiac rhythm.

The secondary outcomes included postoperative inotropic support and mechanical ventilation time. Postoperative inotropic support included the use of dopamine, adrenaline, noradrenaline, milrinone, and dobutamine. Both the proportion and duration of these drugs were recorded. The total duration of inotropic support was the sum of dopamine duration, adrenaline duration, noradrenaline duration, milrinone duration, and dobutamine duration. When postoperative cardiac output was low and cardiovascular performance was bad, dopamine, dobutamine, or milrinone may be infused initially. When an insufficient response was obtained from these drugs, adrenaline was added or substituted. When hypotension existed in the presence of adequate cardiac output, noradrenaline was used. For patients with overt hypothyroidism, levothyroxine treatment started on the first day after surgery.

Continuous variables are expressed as median (25th, 75 th percentile) and were compared in unmatched pairs using the Wilcoxon rank-sum test. Continuous variables in matched pairs were compared using the Wilcoxon signed-rank test. Dichotomous variables are expressed as percentages. The McNemar's test was applied for dichotomous variables in matched pairs data. Risk factors of postoperative complications were analyzed by condition logistic regression, stratified on the matched sets. Factors for which the univariate analysis gave a $P$ value $\leq 0.1$, or of known biologic significance, were included in a logistic multivariate regression model. Odds ratio $(\mathrm{OR})$ and $95 \%$ confidence interval $(\mathrm{CI})$ were calculated.

To account for the intergroup clinical imbalance caused by the selection bias inherent in the nonrandomized nature of the study, a continuous propensity score matching (PSM) analysis was performed. A logistic regression model was built to calculate the probability of each patient and to determine the propensity score. Variables in the model included age, gender, weight, myocardial infarction, diseased vessels, previous percutaneous coronary intervention, hyperlipemia, hypertension, diabetes mellitus, stroke, smoking, chronic obstructive pulmonary disease (COPD), preoperative 
LVEDD, preoperative LVEF, preoperative glomerular filtration rate (GFR), use of $\mathrm{CPB}$, and number of distal anastomoses. A greedy matching algorithm was used to perform the 1:4 match on the logit of the propensity score with a caliper width of 0.2 . Finally, we matched 187 overt hypothyroid patients with 737 euthyroid patients. The balance of the two matched groups was evaluated by standardized mean differences in the matching variables. Usually, a maximum standardized mean difference of 0.1 is considered acceptable.

A $P$ value $<0.05$ was considered to be statistically significant. The statistical analysis was performed using SPSS version 22.0 for Windows (SPSS Inc., Chicago, IL, USA).

\section{RESULTS}

After PSM, the baseline and intraoperative characteristics were similar in the two groups. (Table 1) Before surgery, 168 overt hypothyroid patients received levothyroxine treatment. Overt hypothyroid patients had lower FT4, TT4, free triiodothyronine, total triiodothyronine, and higher TSH levels (all $P<0.001$ ). (Table 2) There were 58 overt hypothyroid patients with normal TSH and 129 overt hypothyroid patients with abnormal TSH before surgery.
In 189 overt hypothyroid patients, the causes of overt hypothyroidism included chronic autoimmune disease (70/189, $37.0 \%)$, thyroidectomy $(43 / 189,22.8 \%)$, benign nodular thyroid disease $(22 / 189,11.6 \%)$, treatment for hyperthyroidism with radioiodine $(33 / 189,17.5 \%)$, hypophysectomy $(1 / 189$, $0.5 \%)$, and unknown causes (20/189, 10.6\%).

The outcomes after CABG are summarized in Table 2. The mechanical ventilation time was longer in overt hypothyroid patients than euthyroid patients (median time: 17 hours vs. 15 hours, $P<0.001$ ). The postoperative stay also was longer in overt hypothyroid patients than euthyroid patients (median time: 7.0 days vs. 6.7 days, $P<0.001$ ). Postoperative LVEDD of overt hypothyroid patients was smaller than that of euthyroid patients $(P=0.04)$, while postoperative LVEF was similar between the two groups. The incidences of impaired wound healing and reintubation were higher in overt hypothyroid patients than euthyroid patients $(11.8 \%$ vs. $0.9 \%, P<0.001 ; 2.1 \%$ vs. $0.4 \%, P=0.03$, respectively). The incidence of the total complications also was higher in overt hypothyroid patients compared with euthyroid patients (39.6\% vs. $30.3 \%, P=0.015)$.

Multivariate analysis showed that overt hypothyroidism was significantly associated with the occurrence of impaired wound healing (OR=12.29, $P<0.001$, Figure 2$)$ reintubation

Table 1. Variables included for the propensity score matching model

\begin{tabular}{|c|c|c|c|c|c|c|}
\hline Age (years), median (Q1, Q3) & $63(57,69)$ & $62(55,67)$ & 0.164 & $63(58,69)$ & $62(57,67)$ & 0.065 \\
\hline Female, n (\%) & $82(43.4 \%)$ & $1465(21.7 \%)$ & 0.437 & $80(42.8 \%)$ & $307(41.7 \%)$ & 0.009 \\
\hline Diseased vessels, median (Q1, Q3) & $3(3,3)$ & $3(3,3)$ & 0.309 & $3(3,3)$ & $3(3,3)$ & 0.039 \\
\hline Previous percutaneous coronary intervention, $\mathrm{n}(\%)$ & $17(9.0 \%)$ & $899(13.3 \%)$ & 0.150 & $17(9.1 \%)$ & $57(7.7 \%)$ & 0.051 \\
\hline Hyperlipemia, n (\%) & $120(63.5 \%)$ & $5155(76.3 \%)$ & 0.265 & $119(63.6 \%)$ & $471(63.9 \%)$ & 0.006 \\
\hline Hypertension, n (\%) & $128(67.7 \%)$ & $4381(64.8 \%)$ & 0.062 & $127(67.9 \%)$ & $491(66.6 \%)$ & 0.030 \\
\hline COPD, n (\%) & $7(3.7 \%)$ & $93(1.4 \%)$ & 0.123 & $7(3.7 \%)$ & $29(3.9 \%)$ & 0.016 \\
\hline Preoperative LVEDD (mm), median (Q1, Q3) & $48(44,50)$ & $48(45,52)$ & 0.263 & $48(44,50)$ & $47(44,50)$ & 0.016 \\
\hline Preoperative LVEF (\%), median (Q1, Q3) & $62(60,65)$ & $61(58,65)$ & 0.196 & $62(60,65)$ & $62(59,65)$ & 0.005 \\
\hline Use of CPB, n (\%) & $80(42.3 \%)$ & $3266(48.3 \%)$ & 0.121 & $80(42.8 \%)$ & $335(45.5 \%)$ & 0.045 \\
\hline Number of distal anastomoses (n), median (Q1, Q3) & $3(3,4)$ & $3(3,4)$ & 0.012 & $3(3,4)$ & $3(3,4)$ & 0.004 \\
\hline Preoperative GFR $(\mathrm{ml} / \mathrm{min})$, median $(\mathrm{Q} 1, \mathrm{Q} 3)$ & $79(66,93)$ & $88(75,99)$ & 0.395 & $79(66,93)$ & $82(69,93)$ & 0.043 \\
\hline
\end{tabular}

COPD, chronic obstructive pulmonary disease; CPB, cardiopulmonary bypass; LVEDD, left ventricular end-diastolic diameter; LVEF, left ventricular ejection

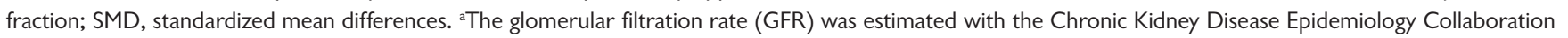
(CKD-EPI) equation. 
(OR=5.71, $P=0.047$, Figure 3), and the total complications (OR=1.31, $P=0.049$, Figure 4). (Figure 2) (Figure 3) (Figure 4) To further evaluate the effect of preoperative thyroid status on postoperative complications, overt hypothyroid patients were divided into two groups: 58 overt hypothyroid patients with normal TSH and the other 129 overt hypothyroid patients with abnormal TSH. (Table 3) After adjusting for age and LVEF by multivariate analysis, the OR of the total complications was $1.10(95 \% \mathrm{CI}, 0.68$ to $1.77, P=0.71)$ in overt hypothyroid patients with normal TSH and 1.43 (95\% CI, 1.04 to $1.98, P=0.03$ ) in overt hypothyroid patients with abnormal TSH compared with euthyroid patients.

Postoperative inotropic supports of drugs are summarized in Table 4. (Table 4) The proportions of the use of dopamine, adrenaline, milrinone, and dobutamine in the overt hypothyroid group were higher than those in the euthyroid group $(75.4 \%$ vs. $67.6 \%, P=0.038 ; 10.7 \%$ vs. $6.1 \%, P=$ $0.028 ; 3.2 \%$ vs. $0.3 \%, P=0.001 ; 4.8 \%$ vs. $1.2 \%, P=0.004$, respectively). The total duration of inotropic support in overt hypothyroid patients was longer than euthyroid patients (median duration: 4 days vs. 3 days, $P=0.003$ ).

\section{DISCUSSION}

This study summarizes our surgical experience of overt hypothyroid patients who underwent CABG surgery. To our knowledge, our study is the largest cohort to compare early postoperative clinical results of overt hypothyroid patients with euthyroid patients. The major finding of this study is

Table 2. Preoperative thyroid function tests and postoperative outcomes

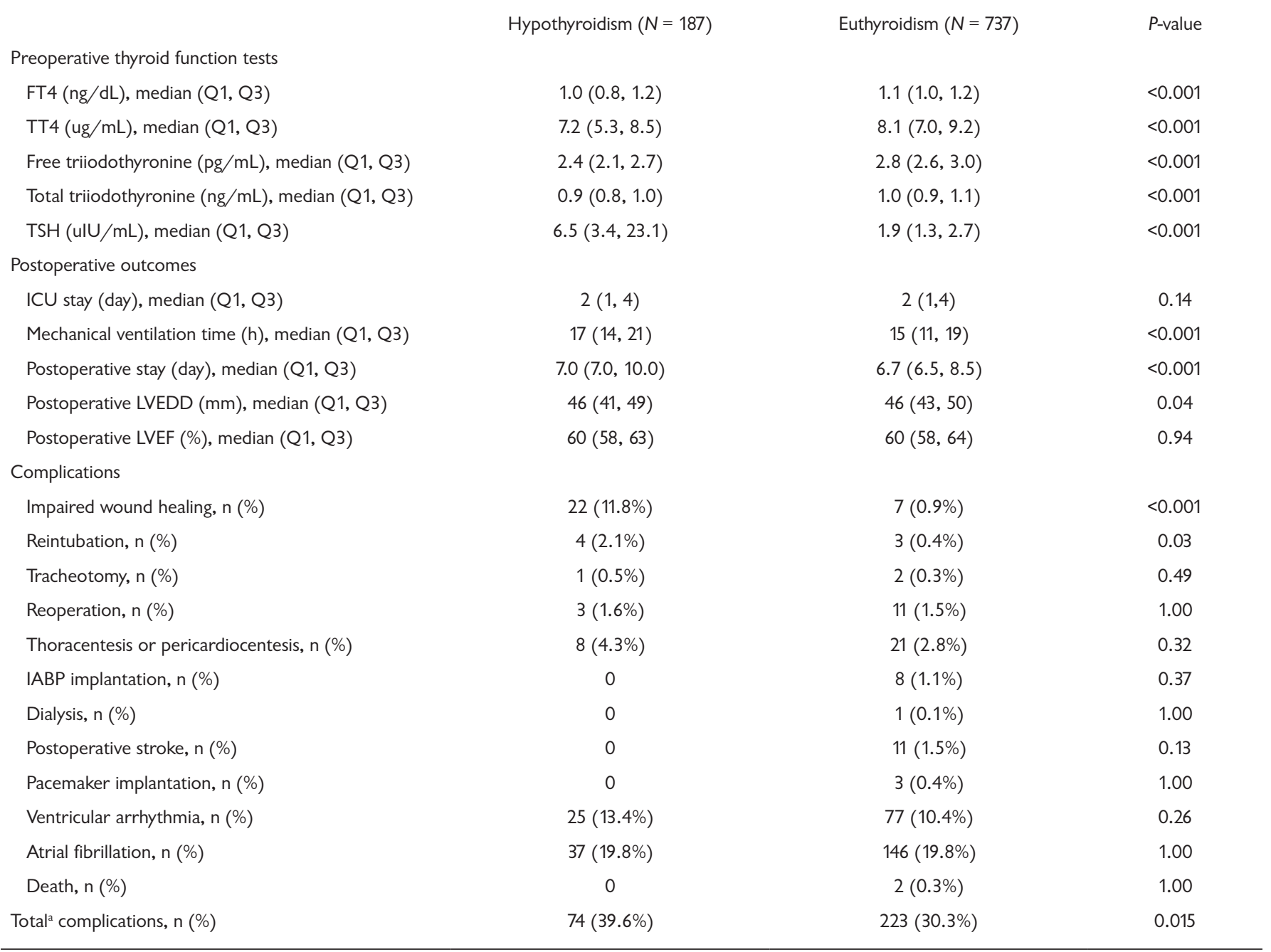

FT4, free thyroxine; LVEDD, left ventricular end-diastolic diameter; LVEF, left ventricular ejection fraction; IABP, intra-aortic balloon pump; ICU, intensive care unit; TSH, thyroid-stimulating hormone; TT4, total thyroxine. aincludes impaired wound healing, reintubation, tracheotomy, reoperation during same hospitalization, thoracentesis or pericardiocentesis, IABP implantation, dialysis, postoperative stroke, pacemaker implantation, ventricular arrhythmia, atrial fibrillation, death. 
that overt hypothyroidism is associated with a higher incidence of impaired wound healing, reintubation, and total postoperative complications, stronger postoperative inotropic support, and longer mechanical ventilation time and hospital stay. Postoperative complications could be reduced by controlling preoperative TSH within the normal range.

In our study, overt hypothyroidism leads to an increased risk of postoperative complications. Many conditions related to overt hypothyroidism may contribute to this result. First, hypothyroidism is associated with increased systemic capillary permeability and disturbances in electrolyte metabolism [Chou 2005]. This could cause fluid retention in interstitial spaces and serous cavities, leading to impaired wound

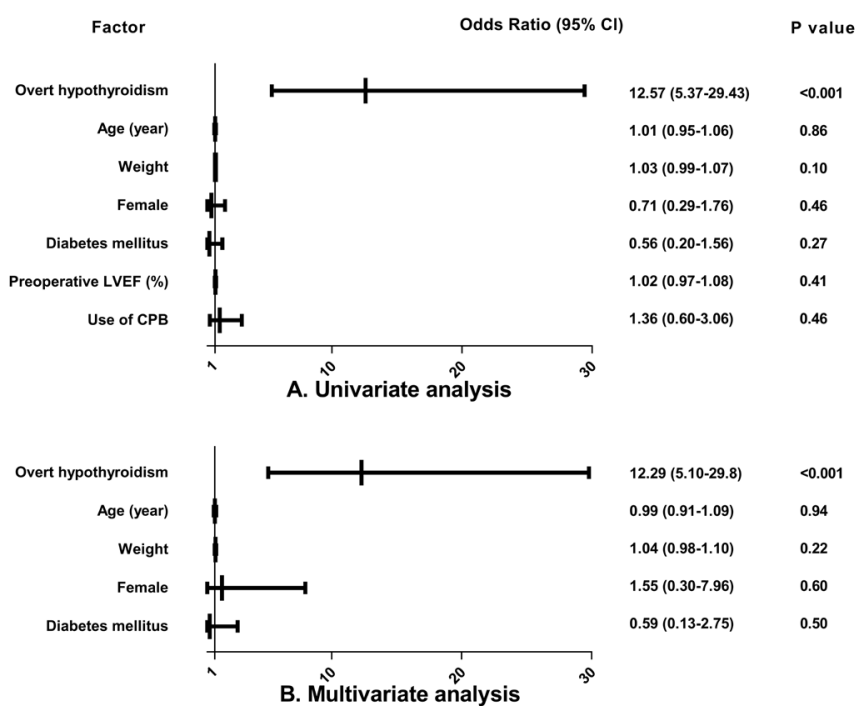

Figure 2. Predictors of impaired wound healing by univariate and multivariate analysis. CPB, cardiopulmonary bypass; LVEF, left ventricular ejection fraction

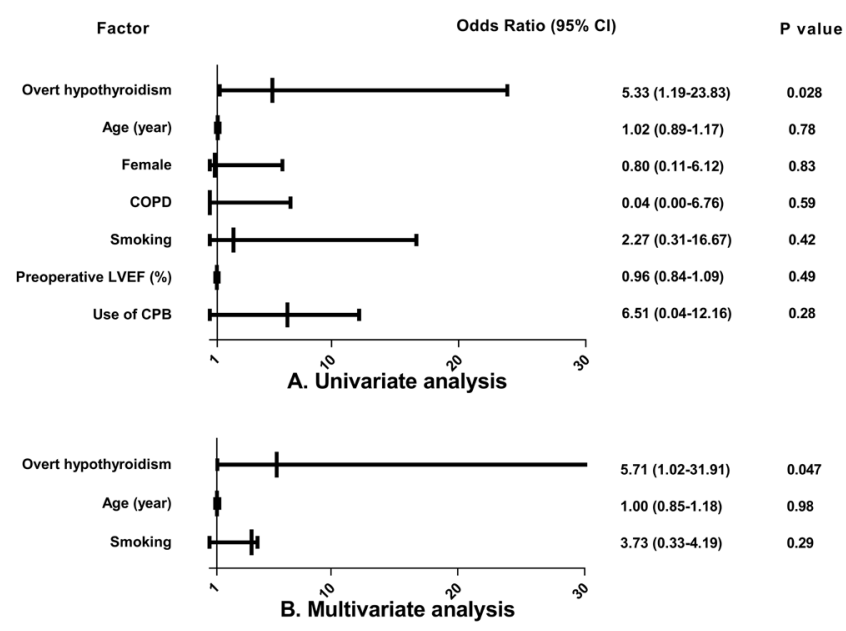

Figure 3. Predictors of reintubation by univariate and multivariate analysis. COPD, chronic obstructive pulmonary disease; CPB, cardiopulmonary bypass; LVEF, left ventricular ejection fraction healing and reoperation. Several studies have been published on pericardial effusions and cardiac tamponade secondary to hypothyroidism [Chou 2005; Ovadia 2007; Patil 2011]. In our study, three hypothyroid patients underwent reoperations because of impaired wound healing and massive pericardial effusions, while 11 euthyroid patients underwent reoperation for controlling bleeding. Second, hypothyroidism could change the structure of connective tissue [Zaki 2013]. Hypothyroid patients had abnormal tissue integrity [Ladenson 1984], which could impair wound healing. Third, hypothyroidism may induce a wide spectrum of alterations in neuromuscular function and lead to muscle dysfunction [Biondi 2008]. Reduced diaphragmatic muscle strength and lower forced vital capacity were found in hypothyroid patients [Cakmak 2011]. In our study, the incidence of reintubation was $2.1 \%$ in overt hypothyroid patients and $0.4 \%$ in euthyroid patients. Fourth, electrocardiographic changes can be seen in hypothyroidism, including sinus bradycardia, a prolonged QTc with increased risk of torsade de pointes ventricular tachycardia, low voltage, and the rare instance of atrioventricular block [Falcone 2014; Grais 2014]. All these factors may lead to an increased risk of postoperative complications in overt hypothyroid patients.

Overt hypothyroid patients also needed stronger and longer postoperative inotropic support than euthyroid patients after CABG, although they had similar preoperative and postoperative LVEFs. The cardiovascular system is a major target of thyroid hormone action. Overt hypothyroidism is associated with impaired cardiac contractility, decreased cardiac output, increased systemic vascular resistance, accelerated atherosclerosis, and coronary artery disease [Grais 2014; Kinugawa 2001; Klein 2007]. In our study, overt hypothyroid patients had smaller LVEDDs than euthyroid patients after surgery, although they shared similar preoperative LVEDDs. This may indicate the association

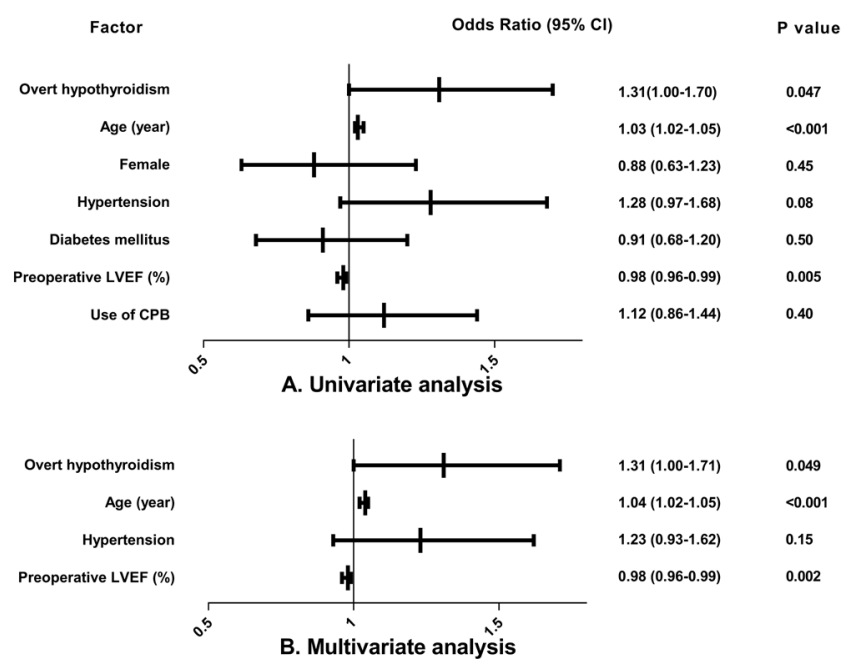

Figure 4. Predictors of the total complications by univariate and multivariate analysis. CPB, cardiopulmonary bypass; LVEF, left ventricular ejection fraction 
between overt hypothyroidism and impairment of left ventricular diastolic function. Hypothyroidism could lead to a dramatic loss of cardiac arterioles, resulting in severe, progressive systolic dysfunction [Tang 2005]. Thyroid hormone deficiency is responsible for an increased risk of heart failure events [Gencer 2012]. In our study, the free triiodothyronine level was lower in overt hypothyroid patients, which was found to be a strong predictor of death and low cardiac output in CABG patients [Cerillo 2014]. All these impairments may lead to stronger and longer postoperative inotropic support in overt hypothyroid patients.

Overt hypothyroid patients with normal preoperative TSH levels have a similar risk of postoperative complications compared with euthyroid patients. Our study was a retrospective exploratory study, and 58 overt hypothyroid patients obtained a normal TSH level before surgery. Among patients with coronary heart disease, preoperative use of levothyroxine will increase oxygen consumption of myocardium and provoke anginal symptoms, which may limit the attainment of euthyroidism. The starting dose is usually low, and the adjustment is slow [Jonklaas 2014]. CABG in overt hypothyroid patients before restoration of euthyroidism was due to severe anginal symptoms or left main coronary artery occlusion. Surgery in these patients is associated with an increased risk of several postoperative complications, which should be anticipated and preemptively managed in the course of their perioperative care.

The incidence of postoperative atrial fibrillation did not differ between overt hypothyroid patients and euthyroid patients. Our incidence of atrial fibrillation was approximate $20 \%$, which was in agreement with previous reports [Kosmidou 2018; Zheng 2016]. Jaimes and his colleagues [Jaimes 2017] reported that hypothyroidism was a risk factor for the onset of postoperative atrial fibrillation in patients undergoing CABG. But the exposed patients in their study included ones only with TSH elevation, and preoperative baseline characteristics were not evenly distributed among the two groups. The perioperative thyroid hormone levels also were not analyzed. Park et al. [Park 2009] reported that subclinical hypothyroidism appeared to increase the development of postoperative atrial fibrillation, but the number of their cohort was small.

Despite the use of various statistical measures (such as PSM and logistic regression) to help control selection bias and confounding factors, the findings should be interpreted cautiously given the relatively small sample size. Also, postoperative thyroid hormone levels were not measured, so that we could not verify the relationship between postoperative

Table 3. Risk of the total complications in hypothyroid patients compared with euthyroid patients after adjusting for age and left ventricular ejection fraction.

\begin{tabular}{lll}
\hline Factor & OR $(95 \% \mathrm{Cl})$ & P-value \\
\hline Hypothyroid patients with normal TSH & $1.10(0.68-1.77)$ & 0.71 \\
Hypothyroid patients with abnormal TSH & $1.43(1.04-1.98)$ & 0.03 \\
\hline
\end{tabular}

$\mathrm{Cl}$, confidence interval; OR, odds ratio; TSH, thyroid-stimulating hormone

Table 4. Postoperative inotropic support

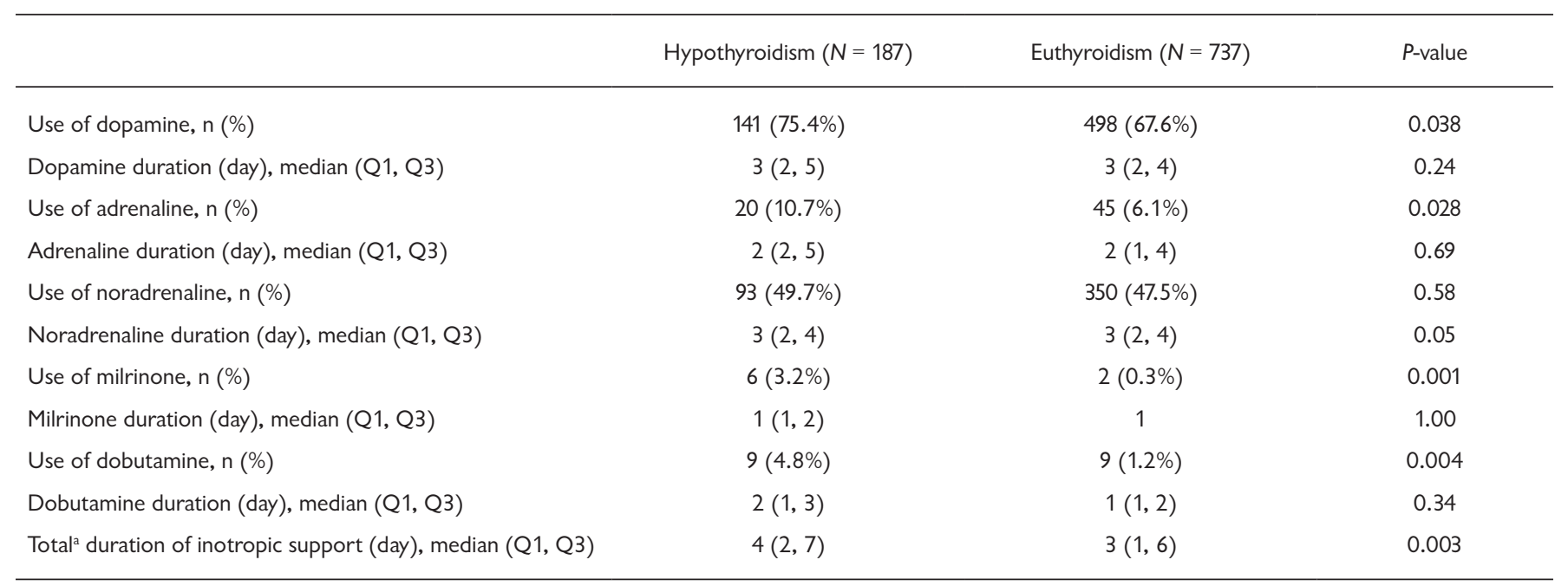

aincludes dopamine duration, adrenaline duration, noradrenaline duration, milrinone duration, and dobutamine duration 
hormone levels and the development of complications. Further large prospective studies with long-term follow up are needed on this issue.

\section{CONCLUSIONS}

CABG in overt hypothyroid patients is associated with a higher incidence of impaired wound healing, reintubation, and total postoperative complications, stronger postoperative inotropic support, and longer mechanical ventilation time and hospital stay. Postoperative complications could be reduced by controlling preoperative TSH within the normal range.

\section{ACKNOWLEDGEMENT}

Funding: This work was supported by the National Key Research and Development Program (2018YFC1311201) from the Ministry of Science and Technology of the People's Republic of China.

\section{REFERENCES}

Biondi B. 2012. Mechanisms in endocrinology: Heart failure and thyroid dysfunction. Eur. J. Endocrinol. 167(5):609-618.

Biondi B, Cooper DS. 2008. The clinical significance of subclinical thyroid dysfunction. Endocr. Rev. 29(1):76-131.

Cakmak G, Saler T, Saglam ZA, et al. 2011. Pulmonary functions in patients with subclinical hypothyroidism. J. Pak. Med. Assoc. 61(10):951-953.

Cerillo AG, Storti S, Kallushi E, et al. 2014. The low triiodothyronine syndrome: a strong predictor of low cardiac output and death in patients undergoing coronary artery bypass grafting. Ann. Thorac. Surg. 97(6):2089-2095.

Chou SL, Chern CH, How CK, Wang LM, Huang CI, Lee CH. 2005. A rare case of massive pericardial effusion secondary to hypothyroidism. J. Emerg. Med. 28(3):293-296.

Falcone C, Matrone B, Bozzini S, et al. 2014. Time-domain heart rate variability in coronary artery disease patients affected by thyroid dysfunction. Int. Heart J. 55(1):33-38.

Gencer B, Collet TH, Virgini V, et al. 2012. Subclinical thyroid dysfunction and the risk of heart failure events: an individual participant data analysis from 6 prospective cohorts. Circulation 126(9):1040-1049.
Grais IM, Sowers JR. 2014. Thyroid and the heart. Am. J. Med. 127(8):691-698.

Jaimes MC, Torrado L, Reyes N, Mackenzie JC, Mallarino J. 2017. Hypothyroidism is a Risk Factor for Atrial Fibrillation after Coronary Artery Bypass Graft. Braz J Cardiovasc Surg 32(6):475-480.

Jonklaas J, Bianco AC, Bauer AJ, et al. 2014. Guidelines for the treatment of hypothyroidism: prepared by the American Thyroid Association task force on thyroid hormone replacement. Thyroid 24(12):1670-1751.

Kinugawa K, Minobe WA, Wood WM, et al. 2001. Signaling pathways responsible for fetal gene induction in the failing human heart: evidence for altered thyroid hormone receptor gene expression. Circulation 103(8):1089-1094.

Klein I, Danzi S. 2007. Thyroid disease and the heart. Circulation 116(15):1725-1735

Kosmidou I, Chen S, Kappetein AP, et al. 2018. New-Onset Atrial Fibrillation After PCI or CABG for Left Main Disease: The EXCEL Trial. J. Am. Coll. Cardiol. 71(7):739-748.

Ladenson PW, Levin AA, Ridgway EC, Daniels GH. 1984. Complications of surgery in hypothyroid patients. Am. J. Med. 77(2):261-266.

Okosieme O, Gilbert J, Abraham P, et al. 2016. Management of primary hypothyroidism: statement by the British Thyroid Association Executive Committee. Clin Endocrinol (Oxf) 84(6):799-808.

Ovadia S, Lysyy L, Zubkov T. 2007. Pericardial effusion as an expression of thyrotoxicosis. Tex Heart Inst J 34(1):88-90.

Park YJ, Yoon JW, Kim KI, et al. 2009. Subclinical hypothyroidism might increase the risk of transient atrial fibrillation after coronary artery bypass grafting. Ann. Thorac. Surg. 87(6):1846-1852.

Patil VC, Patil HV, Agrawal V, Patil S. 2011. Cardiac tamponade in a patient with primary hypothyroidism. Indian J Endocrinol Metab 15(Suppl 2):S144-S146.

Shan Z, Chen L, Lian X, et al. 2016. Iodine Status and Prevalence of Thyroid Disorders After Introduction of Mandatory Universal Salt Iodization for 16 Years in China: A Cross-Sectional Study in 10 Cities. Thyroid 26(8):1125-1130.

Tang YD, Kuzman JA, Said S, Anderson BE, Wang X, Gerdes AM. 2005. Low thyroid function leads to cardiac atrophy with chamber dilatation, impaired myocardial blood flow, loss of arterioles, and severe systolic dysfunction. Circulation 112(20):3122-3130.

Zaki SM, Youssef MF. 2013. Thyroid hormone dysfunctions affect the structure of rat thoracic aorta: a histological and morphometric study. Folia Morphol (Warsz) 72(4):333-339.

Zheng Z, Jayaram R, Jiang L, et al. 2016. Perioperative Rosuvastatin in Cardiac Surgery. N Engl J Med 374(18):1744-1753. 